\title{
Complete genome sequence of Acidimicrobium ferrooxidans type strain (ICP')
}

\author{
Alicia Clum¹, Matt Nolan', Elke Lang', Tijana Glavina Del Rio', Hope Tice', Alex Copeland',

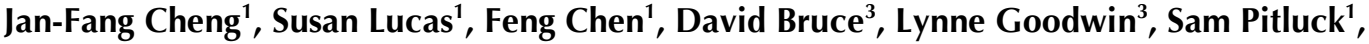 \\ Natalia Ivanova ${ }^{1}$, Konstantinos Mavromatis ${ }^{1}$, Natalia Mikhailova ${ }^{1}$, Amrita Pati ${ }^{1}$, Amy Chen ${ }^{4}$, \\ Krishna Palaniappan ${ }^{4}$, Markus Göker ${ }^{2}$, Stefan Spring ${ }^{2}$, Miriam Land ${ }^{5}$, Loren Hauser ${ }^{5}$, Yun- \\ Juan Chang ${ }^{5}$, Cynthia C. Jeffries ${ }^{5}$, Patrick Chain ${ }^{1,6}$, Jim Bristow ${ }^{1}$, Jonathan A. Eisen ${ }^{1,7}$, Victor \\ Markowitz ${ }^{4}$, Philip Hugenholtz ${ }^{1}$, Nikos C. Kyrpides ${ }^{1}$, Hans-Peter Klenk ${ }^{2}$, and Alla Lapidus ${ }^{1 *}$ \\ ${ }^{1}$ DOE Joint Genome Institute, Walnut Creek, California, USA \\ ${ }^{2}$ DSMZ - German Collection of Microorganisms and Cell Cultures GmbH, Braunschweig, \\ Germany \\ ${ }^{3}$ Los Alamos National Laboratory, Bioscience Division, Los Alamos, New Mexico USA \\ ${ }^{4}$ Biological Data Management and Technology Center, Lawrence Berkeley National Labora- \\ tory, Berkeley, California, USA \\ ${ }^{5}$ Oak Ridge National Laboratory, Oak Ridge, Tennessee, USA \\ ${ }^{6}$ Lawrence Livermore National Laboratory, Livermore, California, USA \\ ${ }^{7}$ University of California Davis Genome Center, Davis, California, USA \\ ${ }^{*}$ Corresponding author: Alla Lapidus
}

Keywords: Moderate thermophile, ferrous-iron-oxidizing, acidophile, Acidomicrobiales.

\begin{abstract}
Acidimicrobium ferrooxidans (Clark and Norris 1996) is the sole and type species of the genus, which until recently was the only genus within the actinobacterial family Acidimicrobiaceae and in the order Acidomicrobiales. Rapid oxidation of iron pyrite during autotrophic growth in the absence of an enhanced $\mathrm{CO}_{2}$ concentration is characteristic for A. ferrooxidans. Here we describe the features of this organism, together with the complete genome sequence, and annotation. This is the first complete genome sequence of the order Acidomicrobiales, and the 2,158,157 bp long single replicon genome with its 2038 protein coding and 54 RNA genes is part of the Genomic Encyclopedia of Bacteria and Archaea project.
\end{abstract}

\section{Introduction}

Acidimicrobium ferrooxidans strain ICPT (DSM $10331=$ NBRC $103882=$ JCM 15462) is the type strain of the species, and was first isolated by Clark and Norris from hot springs in the Krísuvík geothermal area, Iceland [1, 2]. For over fifteen years $A$. ferrooxidans ICPT remained extremely isolated phylogenetically as the sole type strain in the actinobacterial subclass Acidimicrobidae [3] (Figure 1). Only at the time this manuscript was written, Kurahashi et al. [4] and Johnson et al. [5] described three novel type strains representing one novel family, Iamiaceae [4], and two novel genera within the Acidimicrobiales [5]: Iamia majanohamensis (isolated from sea cucumber [4]), Ferromicrobium acidiphilum (from a mine site in North Wales, UK [5]) and Ferrithrix thermotolerans (from Yellowstone National Park, Wyoming, USA [5]). With the exception of $I$. majanohamensis, all these strains live in acidic environments. Here we present a summary classification and a set of features for $A$. ferrooxidans ICPT (Table. 1), together with the description of the complete genomic sequencing and annotation. 
Table 1. Classification and general features of $A$. ferrooxidans ICP ${ }^{\top}$ based on MIGS recommendations [12]

\begin{tabular}{|c|c|c|c|}
\hline MIGS ID & Property & Term & $\begin{array}{l}\text { Evidence } \\
\text { code }\end{array}$ \\
\hline & \multirow{9}{*}{ Current classification } & Domain Bacteria & \\
\hline & & Phylum Actinobacteria & TAS [13] \\
\hline & & Class Actinobacteria & TAS [3] \\
\hline & & Order Acidimicrobiales & TAS [3] \\
\hline & & Suborder Acidimicrobineae & \\
\hline & & Family Acidomicrobiaceae & TAS [3] \\
\hline & & Genus Acidimicrobium & TAS [1] \\
\hline & & Species Acidimicrobium ferrooxidans & TAS [1] \\
\hline & & Type strain ICP & \\
\hline & Gram stain & positive & TAS [1] \\
\hline & Cell shape & rod shaped & TAS [1] \\
\hline & Motility & motile & TAS [1] \\
\hline & Sporulation & nonsporulating & TAS [1] \\
\hline & Temperature range & moderate thermophile, $45-50^{\circ} \mathrm{C}$ & TAS [1] \\
\hline & Optimum temperature & $48^{\circ} \mathrm{C}$ & TAS [1] \\
\hline & Salinity & not reported & \\
\hline \multirow[t]{3}{*}{ MIGS-22 } & Oxygen requirement & aerobic & TAS [1] \\
\hline & Carbon source & $\mathrm{CO}_{2}$ (autotrophic), yeast extract (heterotrophic) & TAS [1] \\
\hline & Energy source & $\begin{array}{l}\text { autotrophic: oxidation of ferrous iron with } \\
\text { oxygen as the electron acceptor; } \\
\text { heterotrophic: yeast extract }\end{array}$ & TAS [1] \\
\hline MIGS-6 & Habitat & $\begin{array}{l}\text { warm, acidic, iron-, sulfur-, or mineral-sulfide } \\
\text { rich environments }\end{array}$ & TAS [1] \\
\hline MIGS-15 & Biotic relationship & free living & NAS \\
\hline \multirow[t]{3}{*}{ MIGS-14 } & Pathogenicity & none & NAS \\
\hline & Biosafety level & 1 & TAS [14] \\
\hline & Isolation & hot springs & TAS [2] \\
\hline MIGS-4 & Geographic location & Krísuvik geothermal area, Iceland & TAS [2] \\
\hline MIGS-5 & Sample collection time & before 1993 & TAS [1] \\
\hline $\begin{array}{l}\text { MIGS-4.1 } \\
\text { MIGS-4.2 }\end{array}$ & Latitude - Longitude & $63.93,-22.1$ & TAS [2] \\
\hline MIGS-4.3 & Depth & not reported & \\
\hline MIGS-4.4 & Altitude & not reported & \\
\hline
\end{tabular}

Evidence codes - IDA: Inferred from Direct Assay (first time in publication); TAS: Traceable Author Statement (i.e., a direct report exists in the literature); NAS: Non-traceable Author Statement (i.e., not directly observed for the living, isolated sample, but based on a generally accepted property for the species, or anecdotal evidence). These evidence codes are from the Gene Ontology project [15]. If the evidence code is IDA the property was directly observed for a live isolate by one of the authors or an expert mentioned in the acknowledgements.

\section{Classification and features}

Members of the species A. ferrooxidans have been isolated or identified molecularly from warm, acidic, iron-, sulphur- or mineral-sulfide-rich environments. Strain TH3, isolated from a copper leaching dump [1], shares 100\% 16S rRNA gene sequence identity with strain ICPT, whose genome sequence is reported here. The moderately thermophilic bacterium N1-45-02 (EF199986) from a spent Canadian copper sulfide heap, and strain Y00168 from a geothermal site in Yellowstone National Park [6] are the only other pure cultivated members of the species. Acidimicrobium species in mixed cultures used for bioleaching were frequently reported [7]. Uncultured clone sequences with significant sequence similarity (>98\%) were observed by Inskeep and colleagues from several hot springs in Yellowstone National Park (e.g. AY882832, 
DQ179032 and others), and from hydrothermally modified volcanic soil at Mount Hood (EU419128). Screening of environmental genomic samples and surveys reported at the NCBI BLAST server indicated no closely related phylotypes (the highest observed sequence identity was 91\%) that can be linked to the species or genus. Several DGGE analyses indicated the presence of members of the genus Acidimicrobium in metal-rich mine waters and geothermal fields around the world.

Figure 1 shows the phylogenetic neighborhood of A. ferrooxidans strain $\mathrm{ICP}^{\mathrm{T}}$ in a $16 \mathrm{~S}$ rRNA based tree. The sequences of the two identical copies of the 16S rRNA genes in the genome differ in 16 positions $(1.1 \%)$ from the previously published 16S rRNA sequence generated from of $A$. ferrooxidans DSM 10331 (U75647). The higher sequence coverage and overall improved level of sequence quality in whole-genome sequences, as compared to ordinary gene sequences, implies that the significant difference between the genome data and the previously reported $16 \mathrm{~S}$
rRNA gene sequence might be due to sequencing errors in the previously reported sequence data.

Cells of strain ICPT are rather small $(0.4 \mu \mathrm{m} \times 1-1.5$ $\mu \mathrm{m})$ Gram-positive rods [1]. Optimal growth occurs at $45-50^{\circ} \mathrm{C}, \mathrm{pH} 2$, with a maximal doubling time of six hours at $48^{\circ} \mathrm{C}$ [1]. Cells are motile during heterotrophic growth on yeast extract. ICPT forms small colonies when grown autotrophically on ferrous iron containing solid medium under air [1]. The closely related strain TH3 differs from the type strain ICP $^{\mathrm{T}}$ only by its tendency to grow in filaments, which has not been observed for strain ICPT $^{T}$ [1]. Strain ICPT $^{\mathrm{T}}$ can be distinguished from members of the genus Sulfobacillus by its lower requirement of $\mathrm{CO}_{2}$ for autotrophic growth [1]. Iron oxidation by ICPT cells was not influenced by supplementation of either glucose nor by increased $\mathrm{CO}_{2}$ concentration [1]. Thin section electron micrographs of $A$. ferrooxidans strains indicate intracellular vesicles when cells were grown on ferrous iron and yeast extract [1] (Figure 2).

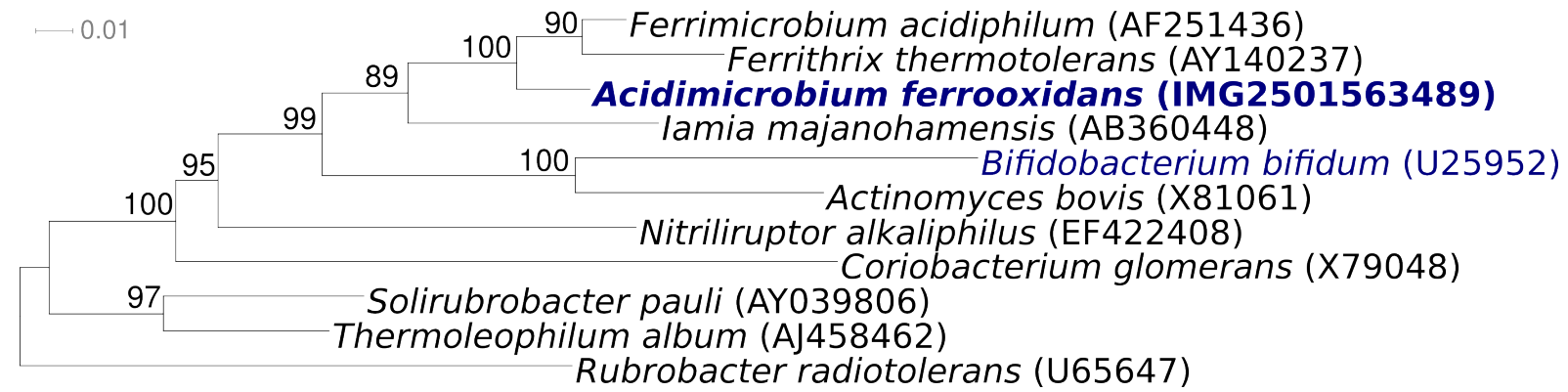

Figure 1. Phylogenetic tree highlighting the position of $A$. ferrooxidans strain $I C \mathrm{P}^{\top}$ relative to all other type strains within the Acidimicrobiales and the type strains of all other orders within the Actinobacteria. The tree was inferred from 1,306 aligned characters [8,9] of the 16S rRNA gene under the maximum likelihood criterion [10] and rooted with Rubrobacteriales. The branches are scaled in terms of the expected number of substitutions per site. Numbers above branches are support values from 1,000 bootstrap replicates if larger than 60\%. Lineages with type strain genome sequencing projects registered in GOLD [11] are shown in blue, published genomes in bold.

\section{Chemotaxonomy}

The murein of $A$. ferrooxidans ICP ${ }^{\mathrm{T}}$ contains mesoDAP, like all other characterized type species from the Acidomicrobineae [3, 4]. It differs from the other characterized Acidomicrobineae strains in MK-9 $\left(\mathrm{H}_{8}\right)$ being the predominant menaquinone, whereas $F$. acidiphilum has $\mathrm{MK}-8\left(\mathrm{H}_{10}\right)$ as the predominant menaquinone [5], and $I$. majanohamensis possesses a mixture $\mathrm{MK}-9\left(\mathrm{H}_{6}\right)$,
MK-9 $\left(\mathrm{H}_{4}\right)$, and MK-9(H) [4]. The major cellular fatty acids of strain ICPT are saturated branched acids: iso- (i-) $\mathrm{C}_{16: 0}(83 \%)$ and anteiso- (ai-) $\mathrm{C}_{17: 0}$ $(8 \%)$ [4], which is more similar to $F$. thermotolerans $\left(90 \% \mathrm{i}-\mathrm{C}_{16: 0}\right)$ and $F$. acidiphilum (64\% i- $\mathrm{C}_{16: 0}$ and $\left.11 \% \mathrm{i}-\mathrm{C}_{14: 0}\right)$ [5], than to $I$. majanohamensis which predominantly possesses straight chain acids $\left(\mathrm{C}_{17: 0}, \mathrm{C}_{16: 0}\right.$ and $\left.\mathrm{C}_{15: 0}\right)[4]$. 


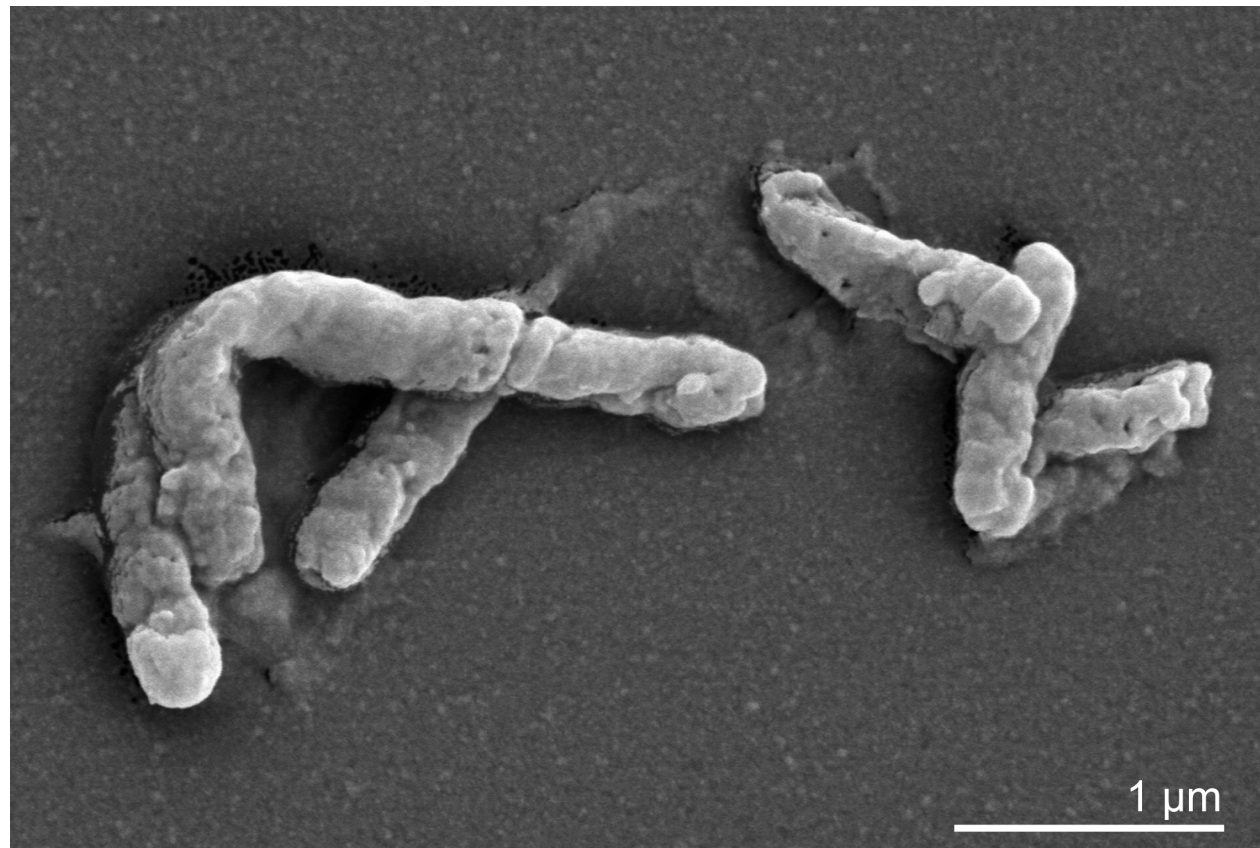

Figure 2. Scanning electron micrograph of $A$. ferrooxidans ICP ${ }^{\top}$ (Manfred Rohde, Helmholtz Centre for Infection Research, Braunschweig)

\section{Genome sequencing and annotation Genome project history}

This organism was selected for sequencing on the basis of each phylogenetic position, and is part of the Genomic Encyclopedia of Bacteria and Archaea project. The genome project is deposited in the Genome OnLine Database [11] and the complete

genome sequence in GenBank (CP001631). Sequencing, finishing and annotation were performed by the DOE Joint Genome Institute (JGI). A summary of the project information is shown in Table 2.

Table 2. Genome sequencing project information

\begin{tabular}{|c|c|c|}
\hline MIGS ID & Property & Term \\
\hline MIGS-31 & Finishing quality & Finished \\
\hline MIGS-28 & Libraries used & $\begin{array}{l}\text { One Sanger library: } 8 \mathrm{~kb} \text { pMCL200 } \\
\text { One } 454 \text { pyrosequence standard library } \\
\text { and one Illumina library }\end{array}$ \\
\hline MIGS-29 & Sequencing platforms & ABI3730, 454 GS FLX, Illumina GA \\
\hline MIGS-31.2 & Sequencing coverage & $6.8 \times$ Sanger; $52.9 \times$ pyrosequence \\
\hline MIGS-30 & Assemblers & Newbler, Arachne \\
\hline \multirow[t]{5}{*}{ MIGS-32 } & Gene calling method & Prodigal \\
\hline & INSDC / Genbank ID & СР001631 \\
\hline & Genbank Date of Release & not available \\
\hline & GOLD ID & Gc01023 \\
\hline & Database: IMG-GEBA & 2501533204 \\
\hline \multirow[t]{2}{*}{ MIGS-13 } & Source material identifier & DSM 10331 \\
\hline & Project relevance & Tree of Life, GEBA \\
\hline
\end{tabular}




\section{Growth conditions and DNA isolation}

A. ferrooxidans strain ICPT, DSM 10331, was grown in DSMZ medium 709 (Acidimicrobium Medium) at $45^{\circ} \mathrm{C}$. DNA was isolated from 1-1.5 g of cell paste using Qiagen Genomic 500 DNA Kit (Qiagen, Hilden, Germany) with a modified protocol for cell lysis containing an additional $200 \mu$ lysozyme and doubled (1 hour) incubation at $37^{\circ} \mathrm{C}$.

\section{Genome sequencing and assembly}

The genome was sequenced using a combination of Sanger, 454 and Illumina sequencing platforms. All general aspects of library construction and sequencing performed at the JGI can be found at the JGI web site. 454 Pyrosequencing reads were assembled using the Newbler assembler version 1.1.02.15 (Roche). Large Newbler contigs were broken into 2356 overlapping fragments of $1000 \mathrm{bp}$ and entered into assembly as pseudoreads. The sequences were assigned quality scores based on Newbler consensus q-scores with modifications to account for overlap redundancy and adjust inflated q-scores. A hybrid 454/Sanger assembly was made using the Arachne assembler. Possible mis-assemblies were corrected and gaps between contigs were closed by custom primer walks from sub-clones or PCR products. 118 Sanger finishing reads were produced. Illumina reads were used to improve the final consensus quality using an in-house developed tool (the
Polisher). The error rate of the completed genome sequence is less than 1 in 100,000 . Together, the combination of the Sanger and 454 sequencing platforms provided $59.7 \times$ coverage of the genome.

\section{Genome annotation}

Genes were identified using Prodigal [16] as part of the Oak Ridge National Laboratory genome annotation pipeline, followed by a round of manual curation using the IGI GenePRIMP pipeline [17]. The predicted CDSs were translated and used to search the National Center for Biotechnology Information (NCBI) nonredundant database, UniProt, TIGRFam, Pfam, PRIAM, KEGG, COG, and InterPro databases. Additional gene prediction analysis and functional annotation was performed within the Integrated Microbial Genomes (IMG-ER) platform [18].

\section{Genome properties}

The genome is 2,158,157 bp long and comprises one main circular chromosome with a $68.3 \%$ GC content (Table 3 and Figure 3). Of the 2,092 genes predicted, 2038 were protein coding genes, and 54 RNAs. Seventy four pseudogenes were also identified. A total of $75.7 \%$ of the genes were assigned a putative function while the remaining ones were annotated as hypothetical proteins. The distribution of genes into COGs functional categories is presented in Table 4 .

Table 3. Genome Statistics

\begin{tabular}{lrr}
\hline Attribute & Value & \% of Total \\
\hline Genome size (bp) & $2,158,157$ & $100.00 \%$ \\
DNA Coding region (bp) & $1,988,736$ & $92.15 \%$ \\
DNA G+C content (bp) & $1,473,791$ & $68.29 \%$ \\
Number of replicons & 1 & \\
Extrachromosomal elements & 0 & \\
Total genes & 2092 & $100.00 \%$ \\
RNA genes & 54 & $2.58 \%$ \\
rRNA operons & 2 & \\
Protein-coding genes & 2038 & $97.42 \%$ \\
Pseudo genes & 74 & $3.54 \%$ \\
Genes with function prediction & 1584 & $75.72 \%$ \\
Genes in paralog clusters & 1969 & $9.37 \%$ \\
Genes assigned to COGs & 1526 & $72.94 \%$ \\
Genes assigned Pfam domains & 1603 & $76.63 \%$ \\
Genes with signal peptides & 591 & $28.25 \%$ \\
Genes with transmembrane helices & 436 & $20.84 \%$ \\
CRISPR repeats & 2 & \\
\hline
\end{tabular}




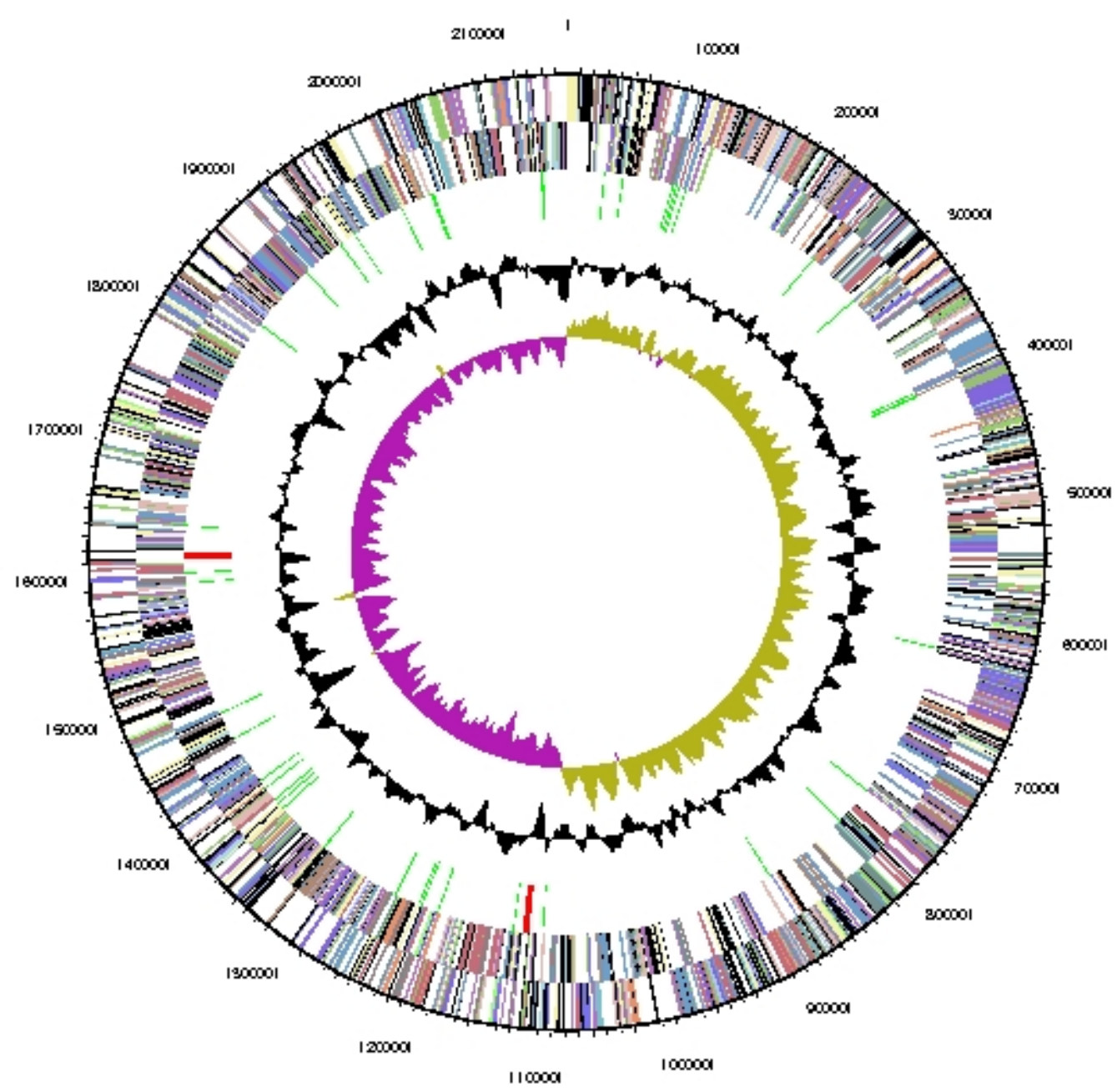

Figure 3. Graphical circular map of the genome. From outside to the center: Genes on forward strand (color by COG categories), Genes on reverse strand (color by COG categories), RNA genes (tRNAs green, rRNAs red, other RNAs black), GC content, GC skew.

Table 4. Number of genes associated with the 21 general COG functional categories

\begin{tabular}{lrrl} 
Code & Value & $\%$ & \multicolumn{2}{l}{ Description } \\
\hline J & 134 & 6.6 & Translation, ribosomal structure and biogenesis \\
A & 1 & 0.0 & RNA processing and modification \\
K & 94 & 4.6 & Transcription \\
L & 119 & 5.8 & Replication, recombination and repair \\
B & 1 & 0.0 & Chromatin structure and dynamics \\
D & 24 & 1.2 & Cell cycle control, mitosis and meiosis \\
Y & 0 & 0.0 & Nuclear structure \\
V & 22 & 1.1 & Defense mechanisms \\
T & 73 & 3.6 & Signal transduction mechanisms \\
M & 84 & 4.1 & Cell wall/membrane biogenesis \\
N & 31 & 1.5 & Cell motility \\
Z & 0 & 0.0 & Cytoskeleton \\
W & 0 & 0.0 & Extracellular structures \\
\hline
\end{tabular}




\begin{tabular}{lrrl}
\multicolumn{3}{l}{ Table 4. Number of genes associated with the 21 general COG functional categories (cont.) } \\
\hline Code & Value & \% & \multicolumn{2}{l}{ Description } \\
\hline O & 70 & 3.4 & Posttranslational modification, protein turnover, chaperones \\
C & 149 & 7.3 & Energy production and conversion \\
G & 87 & 4.3 & Carbohydrate transport and metabolism \\
E & 172 & 8.4 & Amino acid transport and metabolism \\
F & 54 & 2.6 & Nucleotide transport and metabolism \\
H & 110 & 5.4 & Coenzyme transport and metabolism \\
I & 86 & 4.2 & Lipid transport and metabolism \\
P & 60 & 2.9 & Inorganic ion transport and metabolism \\
Q & 34 & 1.7 & Secondary metabolites biosynthesis, transport and catabolism \\
R & 151 & 7.4 & General function prediction only \\
S & 98 & 4.8 & Function unknown \\
- & 512 & 25.1 & Not in COGs \\
\hline
\end{tabular}

\section{Acknowledgements}

We would like to gratefully acknowledge the help of Petra Aumann for growing A. ferrooxidans cultures and Susanne Schneider for DNA extraction and quality analysis (both at DSMZ). This work was performed under the auspices of the US Department of Energy Office of Science, Biological and Environmental Research Program, and by the University of California,

\section{References}

1. Clark DA, Norris PR. Acidimicrobium ferrooxidans gen. nov., sp. nov.: mixed-culture ferrous iron oxidation with Sulfobacillus species. Microbiology 1996; 142:785-790 doi:10.1099/00221287-142-4-785

2. Norris PR, Owen JP. Mineral sulphide oxidation by enrichment cultures of novel thermoacidophilic bacteria. FEMS Microbiol Rev 1993; 11:51-55 doi:10.1111/j.15746976.1993.tb00266.x

3. Stackebrandt E, Rainey F, Ward-Rainey N. Proposal for a new hierarchic classification system, Actinobacteria classis nov. Int / Syst Bacteriol 1997; 47:479-491 doi:10.1099/00207713-47-2-479

4. Stamatakis A, Hoover P, Rougemont J. A rapid bootstrap algorithm for the RAxML Web servers. Syst Biol 2008; 57:758-771 PMID:18853362 doi:10.1080/10635150802429642

5. Kurahashi M, Fukunaga Y, Sakiyama Y, Harayama S, Yokota A. lamia majanohamensis gen. nov., sp. nov., an actinobacterium isolated from sea cucumber Holothuria edulis,
Lawrence Berkeley National Laboratory under contract No. DE-AC02-05CH11231, Lawrence Livermore National Laboratory under Contract No. DE-AC5207NA27344, and Los Alamos National Laboratory under contract No. DE-AC02-06NA25396, as well as German Research Foundation (DFG) INST 599/1-1.

and proposal of lamiaceae fam. nov. Int J Syst Evol Microbiol 2009; 59:869-873 PMID:19329622 doi:10.1099/ijs.0.005611-0

6. Johnson DB, Bacelar-Nicolau P, Okibe N, Thomas A, Hallberg KB. Ferrimicrobium acidiphilum gen. nov., sp. nov. and Ferrithrix thermotolerans gen. nov., sp. nov.: heterotrophic, iron-oxidizing, extremely acidophilic actinobacteria. Int / Syst Evol Microbiol 2009; 59:1082-1089 PMID:19406797 doi:10.1099/ijs.0.65409-0

7. Johnson DB, Okibe N, Roberto FF. Novel thermo-acidophilic bacteria isolated from geothermal sites in Yellowstone National Park: physiological and phylogenetic characteristics. Arch Microbiol 2003; 180:60-68 PMID:12802481 doi:10.1007/s00203-003-0562$\underline{3}$

8. Cleaver AA, Burton NP, Norris PR. A novel Acidimicrobium species in continuous cultures of moderately thermophilic, mineral-sulfideoxidizing acidophiles. Appl Environ Microbiol 2007; 73:4294-4299 PMID:17468267 doi:10.1128/AEM.02658-06 
9. Castresana J. Selection of conserved blocks from multiple alignments for their use in phylogenetic analysis. Mol Biol Evol 2000; 17:540-552 PMID:10742046

10. Lee C, Grasso C, Sharlow MF. Multiple sequence alignment using partial order graphs. Bioinformatics 2002; 18:452-464 PMID:11934745 doi:10.1093/bioinformatics/18.3.452

11. Liolios K, Mavromatis K, Tavernarakis N, Kyrpides NC. The Genomes On Line Database (GOLD) in 2007: status of genomic and metagenomic projects and their associated metadata. Nucleic Acids Res 2008; 36:D475479 PMID:17981842 doi:10.1093/nar/gkm884

12. Field D, Garrity G, Gray T, Morrison N, Selengut J, Sterk P, Tatusova T, Thomson N, Allen MJ, Angiuoli SV, et al. The minimum information about a genome sequence (MIGS) specification. Nat Biotechnol 2008; 26:541547 PMID:18464787 doi:10.1038/nbt1360

13. Cavalier-Smith T. The neomuran origin of archaebacteria, the negibacterial root of the universal tree and bacterial megaclassification.
Int J Syst Evol Microbiol 2002; 52:7-76

PMID:11837318

14. Anonymous. Biological Agents: Technical rules for biological agents. <www.baua.de $>$.

15. Ashburner M, Ball CA, Blake JA, Botstein D, Butler H, Cherry JM, Davis AP, Dolinski K, Dwight SS, Eppig JT, et al. Gene ontology: tool for the unification of biology. The Gene Ontology Consortium. Nat Genet 2000; 25:25-29 PMID: 10802651 doi:10.1038/75556

16. Anonymous. Prodigal Prokaryotic Dynamic Programming Genefinding Algorithm. Oak Ridge National Laboratory and University of Tennessee $2009<$ compbio.ornl.gov/prodigal/>

17. Pati. GenePRIMP: A Gene Prediction Improvement Pipeline for microbial genomes. (Submitted).

18. Markowitz V, Mavromatis K, Ivanova N, Chen I-M, Chu K, Palaniappan K, Szeto E, Anderson I, Lykidis A, Kyrpides N. Expert Review of Functional Annotations for Microbial Genomes. (Submited 2009). 